\title{
EFEITOS DO FOGO SOBRE ALGUMAS VARIÁVEIS MICROMETEOROLÓGICAS EM UMA FLORESTA DE BRACATINGA (Mimosa scabrella, Benth.), NO MUNICÍPIO DE COLOMBO, PR ${ }^{1}$
}

\author{
Leocadio Grodzki ${ }^{2}$ \\ Ronaldo Viana Soares ${ }^{3}$ \\ Antonio Carlos Batista ${ }^{4}$ \\ E Paulo Henrique Caramori ${ }^{5}$
}

\begin{abstract}
RESUMO
O sistema agroflorestal da bracatinga utiliza queima após o corte e retirada da madeira, dando lugar à semeadura de espécies agrícolas. A queima controlada altera a temperatura do ar e do solo. A mudança de refletividade da superfície é mais rápida que dos reflorestamentos próximos. A transformação das folhas e galhos secos em cinza após a queima, faz com que haja mudanças do albedo, alterando o balanço energético. Os resultados mostram temperaturas do ar de $600^{\circ} \mathrm{C}$ por $20-40$ segundos a $1 \mathrm{~cm}$ do solo e de 100 a $300^{\circ} \mathrm{C}$ a 60 e $160 \mathrm{~cm}$ do solo, respectivamente, durante 1 minuto. Temperaturas de $100^{\circ} \mathrm{C}$ ao nível do solo residiram por mais de 3 minutos. A temperatura do solo não foi afetada a $2,5 \mathrm{~cm}$ de profundidade. Durante a queima, a temperatura se elevou em $1^{\circ} \mathrm{C}$. $\mathrm{O}$ albedo de 0,24 antes da queima, passou para 0,21 logo após a queima. Após 60 dias, o albedo voltou a 0,24 devido a recomposição da vegetação.

Palavras chave: temperaturas do ar e do solo, queima de bracatinga, albedo, área degradada, floresta
\end{abstract}

\section{FIRE EFECTS ON SOME MICROMETEOROLOGICAL VARIABLES IN A BRACATINGA (MimOSA scabrella, Benth.) FOREST, COLOMBO, PR}

\begin{abstract}
The bracatinga agriculture-forest systems adopted by farmers consists on burning the residues after wood's harvesting prior to sowing the crops. This procedure is repeated each 6 to 8 years in the same area. The prescribed burning changes air and soil temperatures. Changes in reflectivity are faster then in the surrounding forest areas. Transforming leaves and branches into ashes after burning changes the albedo of the surface, altering the energetic balance. Results showed air temperatures of $600^{\circ} \mathrm{C}$ during 20 to 40 seconds, $1 \mathrm{~cm}$ above the soil surface, and 100 to $300^{\circ} \mathrm{C}$ at 60 and $160 \mathrm{~cm}$ above the soil surface, during 1 minute. Temperatures over $100^{\circ} \mathrm{C}$ on the soil surface were observed for more than 3 minutes. Soil temperature was not affected at $2.5 \mathrm{~cm}$ depth; during burning, the temperature raised only $1^{\circ} \mathrm{C}$. The surface albedo that was 0,24 before the burning changed to 0,21 after burning and returned to 0.24 sixty days after the burning due to the vegetation regeneration.
\end{abstract}

Key words: air and soil temperatures, burning, bracatinga, albedo, degraded area, forest

\section{INTRODUÇÃO}

A bracatinga ocupa, somente na região de Metropolitana de Curitiba, uma área aproximada de 85.000 ha. A sua exploração se dá em função da demanda por lenha, carvão, construção civil, envaramento de olerícolas bem como para empregos mais nobres como a fabricação de móveis. O sistema agroflorestal que vem sendo adotado pelos agricultores é o de queima dos resíduos florestais após o corte e retirada da madeira, para a semeadura de espécies agrícolas, situação que se repete a cada 6 a 8 anos. A queima é conduzida de forma controlada nas áreas que serão queimadas, as quais não ultrapassam 6 ha.

\footnotetext{
${ }^{1}$ Parte integrante da tese do primeiro autor, realizada junto ao Departamento de Conservação da Natureza da Escola de Floresta da UFPR, para obtenção do título de Doutor em Ciências Florestais

${ }^{2}$ Dr., Pesquisador do IAPAR/SIMEPAR, Curitiba, Caixa Postal 19100, CEP 81531-990, E-mail: lgrodzki@ufpr.br

${ }^{3}$ PhD., Professor titular da Escola de Floresta da UFPR, Av. Lothario Meissner 3400, Curitiba, CEP 80210-170, E-mail: rvsoares@florestas.ufpr.br.

${ }^{4}$ Dr., Professor da Escola de Floresta da UFPR, Av. Lothario Meissner 3400, Curitiba, CEP 80210-170, E-mail: batista@florestas.ufpr.br

${ }_{5}^{5}$ PhD., Pesquisador do IAPAR, Londrina, Caixa Postal 481, CEP 86001-970, E-mail: caramori@pr.gov.br
} 
Estas áreas são em sua maioria contornadas por florestamentos e madeira que foi retirada. Embora o processo de queima se desenvolva rapidamente, implica em uma mudança drástica do meio ambiente.

Esta situação permanece por um intervalo de aproximadamente 60 dias quando então o repovoamento do solo com espécies nativas desencadeia um processo de reestruturação do meio. O colorido cinzento do local passa para tons do verde, muito embora a falta da cobertura original opere grandes transformações no microclima local, tais como a temperatura do ar e do solo e a refletividade da superfície, acarretando diferentes acúmulos de energia do meio.

O albedo de uma superfície, que por definição é a razão entre a radiação solar refletida e a radiação solar incidente, tende ter uma simetria em áreas homogêneas, aumentando seu valor quanto mais próximo do nascer ou do pôr do sol, devido a maior refletividade da superfície nestas horas. Por outro lado, em determinados dias essa simetria entre a variação do ângulo zenital e a do albedo pode não existir, como em dias de vento (Song, 1998). A inclinação do dossel pode mudar a reflexão assimetricamente, atingindo valores diferenciados em até $10 \%$ entre a manhã e a noite. Resultados também diferenciados entre os períodos matutinos e vespertinos foram encontrados por Gielow et al. (1998). Dias et al. (1998) mostrou valores de albedo em uma cultura de soja, variando de um máximo matinal de 0,30 , passando por um mínimo de 0,22 por volta das 15 horas e aumentando para 0,65 ao pôr do sol. As medidas de albedo foram amplamente discutidas em vários trabalhos recentes no Brasil, como mostram Dias et al. (1998), Okawa et al. (1998), e Okawa et al. (1999), porém em áreas com cultivo de plantas agrícolas ou em áreas cobertas com água. No entanto a situação é diferente em áreas florestadas com bracatinga, seja pela cobertura vegetal ou pela topografia do terreno. Esta situação é intensamente diferenciada, quando se formam os mosaicos de áreas povoadas de bracatinga ao lado de ilhas de floresta derrubada e queima dos resíduos florestais.

Muitas são as referências sobre as temperaturas das chamas próximo ao solo. De acordo com Wright e Bailey (1982) em áreas de campo podem ser atingidas temperaturas da ordem de $800^{\circ} \mathrm{C}$, embora outros trabalhos se reportem a temperaturas menores (Ito e Izumi, 1960).

A literatura brasileira é ainda escassa quando se trata de avaliar os fenômenos físicos que envolvem a vegetação natural e principalmente com relação a bracatinga.

Alguns trabalhos foram desenvolvidos em outros ecossistemas, como o de Coutinho (1990) e Miranda et al. (1996). Em queima de campo sujo de cerrado a temperatura do ar no ponto máximo das chamas atingiu valores da ordem de $899^{\circ} \mathrm{C}$, próximo ao Distrito Federal (Coutinho,1990). Miranda et al. (1996) em estudos também no cerrado, encontraram temperaturas máximas do ar entre 604 e $752^{\circ} \mathrm{C}$ a $60 \mathrm{~cm}$ de altura do solo. A $1 \mathrm{~cm}$, os valores variaram de 578 a $672^{\circ} \mathrm{C}$ e a $160 \mathrm{~cm}$ de 346 a $700^{\circ} \mathrm{C}$. Esta variabilidade esteve associada às condições climáticas que antecederam a queima. Segundo os autores, a permanência da temperatura acima de $60^{\circ} \mathrm{C}$ foi no máximo de 150 segundos, porém o suficiente para causar a morte das folhas expostas ao fluxo de ar quente.

Embora o processo de queima controlada dos restos de um bracatingal se processe rapidamente, seus efeitos são sentidos também nos primeiros centímetros de solo. No solo, vai depender do tempo de residência e da manta orgânica que permanecer úmida protegendo sua superfície. Segundo Ralston e Hatchell (1971), os efeitos da temperatura do solo são menores numa queima controlada do que num incêndio. Na Flórida e sul do Mississipi, queimas contra o vento geraram mais de $52^{\circ} \mathrm{C}$ por mais de 15 minutos entre 3 a $6 \mathrm{~cm}$ de profundidade e a 2 a 3 minutos com $121^{\circ} \mathrm{C}$. DE Bano (1977) cita temperaturas acima de $100^{\circ} \mathrm{C}$ na superfície do solo durante 15 minutos. A $2,5 \mathrm{~cm}$ de profundidade, as temperaturas máximas foram de 90 a $195^{\circ} \mathrm{C}$ em várias queimas. Coutinho (1990) encontrou temperaturas de $74^{\circ} \mathrm{C}$ próximas a superfície do solo em queimas de campo sujo de cerrado e Neves e Miranda (1996) detectaram temperaturas de $52,8^{\circ} \mathrm{C}$ a $1 \mathrm{~cm}$ de profundidade, no mesmo ecossistema. É evidente que as condições microclimáticas no momento da queima assim como a umidade e quantidade de combustível, serão as condições preponderantes para a eficiência do processo. 
O estudo proposto se desenvolveu em condições naturais de queima efetuada pelo agricultor sobre uma quantidade de combustível estimada em 24,8t/ha.

\section{OBJETIVOS}

Procurou-se determinar micrometeorologicamente as variáveis climáticas:

- albedo antes, logo após a queima e 60 dias após em área de queima dos restos de cultivo de bracatinga;

- comparar as medidas de albedo na área desmatada com uma área similar, adjacente, florestada com bracatinga;

- medir a temperatura do ar, em três alturas, durante a passagem do fogo, na queima dos resíduos de bracatinga;

- medir a temperatura do solo durante a passagem do fogo, na queima dos resíduos de bracatinga.

\section{MATERIAL E MÉTODOS}

Foram instalados dois postes metálicos para as medidas do albedo. Um deles foi instalado dentro de um povoamento de bracatinga de aproximadamente 3 anos de idade e o outro em local adjacente, porém em área de recente derrubada e extração de toras de bracatinga. Em ambos os postes foram instalados piranômetros Eppley na altura de 6 metros, para as medidas de radiação solar incidente e refletida, na faixa do visível e infravermelho próximo. Os dados obtidos a cada 5 minutos foram registrados num "Datalogger" 21X da Campbell Scientific. Na área de queima dos resíduos de bracatinga e para a montagem dos sensores de temperatura, utilizou-se de um pequeno poste de $200 \mathrm{~cm}$ de altura onde foram instalados os termopares de chromel/alumel nas alturas de $1 \mathrm{~cm} ; 60 \mathrm{~cm}$ e $160 \mathrm{~cm}$ do solo. Os termopares foram isolados entre si e presos ao poste através de isoladores cerâmicos. Procedeu-se a aquisição dos dados em um "Datalogger" 21X, programado para coleta a cada 2 e 5 segundos. As temperaturas de queima foram monitoradas até 30 minutos após a passagem do fogo pelos sensores. Para as medidas de temperatura de solo foi utilizado um termopar de cobre/constantã enterrado a uma profundidade de $2,5 \mathrm{~cm}$ e as coletas de dados foram feitas também em um "datalogger" 21X da Campbell Scientifc.

\section{RESULTADOS E DISCUSSÃO}

Albedo: Os resultados da área desmatada são mostrados na fig.1. O albedo médio variou de 0,21 a 0,24 entre os dias $27 / 08$ e $03 / 09$. A variabilidade dos valores ao longo dos dias pode ser atribuída à variação da arquitetura das folhas das plantas herbáceas que cobriam boa parte da superfície, devido a mudança de posição das folhas em função do vento e variação do ângulo zenital. Cabe salientar que embora fosse uma área desmatada, havia muitas herbáceas e brotações de tocos de árvores do secundário vegetando sobre o solo. Os valores do albedo tenderam ao infinito nos extremos do dia, sendo mínimos por volta das 15 horas, como os encontrados por Dias et al. (1998).

Com a passagem do fogo no dia 02/09, houve um decréscimo no albedo, função provável da fumaça formada por volta das 14 horas, que se estendeu de forma incerta até o final da tarde e que contribuiu para a variabilidade encontrada no albedo neste dia. No dia $03 / 09$, quando se processou a segunda etapa da queima, os valores do albedo decresceram em função da mudança da reflexão da superfície, coberta em grande parte por cinza e carvão. Um mês após a queima (03 a 09/10), o albedo médio decresceu para 0,20 (figura 2), situação semelhante ao encontrado por Dias et al. (1996), para campo limpo de cerrado.

Estimou-se em cerca de $20 \%$ a cobertura verde sobre a área de cinzas e carvão. Trinta dias após a passagem do fogo na área de estudo, já havia a rebrota de tocos de espécies nativas bem como brotações de herbáceas e lianas além da germinação da própria bracatinga. Aos sessenta dias após a queima dos resíduos florestais, a cobertura vegetal se completou cobrindo todo o solo. $\mathrm{O}$ albedo médio ficou em 0,24.

Para a área coberta com bracatinga, o valor médio do albedo entre os dias 28/08 e 03/09 (Figura 3) ficou em 0,16, indicando uma menor refletividade ao fim do dia em função do maior ângulo zenital e conseqüentemente menor penetração da luz no dossel. Entre os dias 03 e 09/10 (figura 4), os valores médios cresceram ficando em 0,19 decrescendo para 0,17 sessenta dias após, valores esses dentro da faixa encontrada por alguns autores (Rose, 1971; Monteith, 1973). 


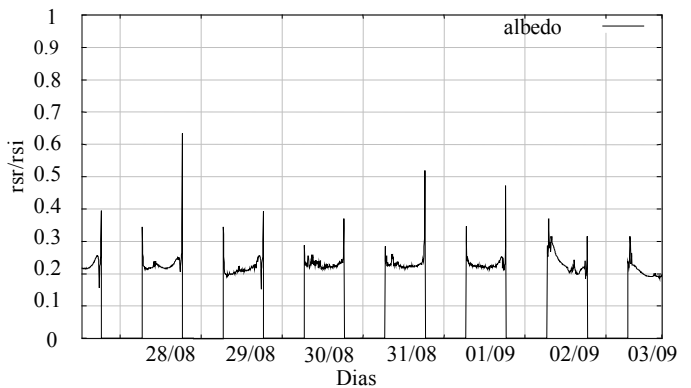

Figura 1: Albedo na área desmatada (28/08 a 03/09 Figure 1: Albedo in the deforested area (28/08 to 03/09

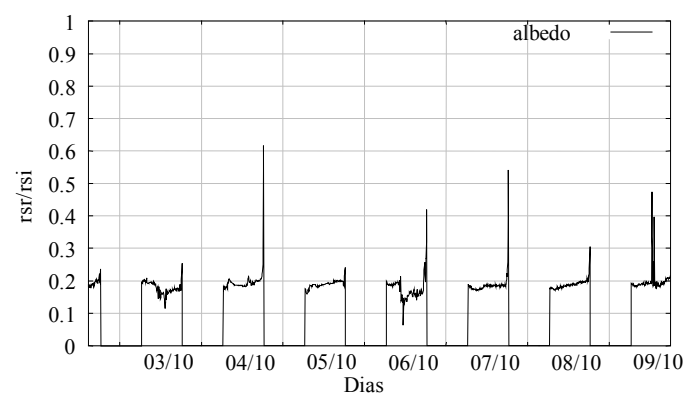

Figura 2: Albedo na área desmatada (03 a 09/10) Figure 2: Albedo in the deforested área (03 a 09/10)

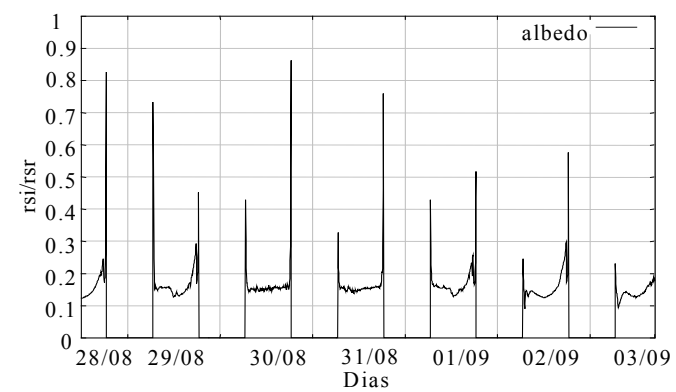

Figura 3: Albedo na área de bracatinga (28/08 a 03/09)

Figure 3: Albedo in the bracatinga area (28/08 a 03/09)

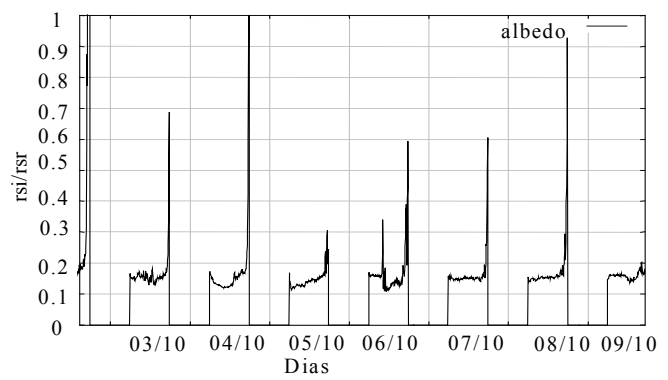

Figura 4: Albedo na área de bracatinga (03 a 09/10)

Figure 4: Albedo in the bracatinga area (03 a 09/10)
Temperatura do ar: A fig. 5 mostra que a $1 \mathrm{~cm}$ as temperaturas atingiram valores ao redor de $600^{\circ} \mathrm{C}$ por um período aproximado de 20 segundos. Quando se comparam estes valores máximos com os obtidos a $160 \mathrm{~cm}$ de altura, observa-se uma defasagem no tempo, função provável da inclinação das chamas em razão da turbulência do gradiente térmico e da velocidade do vento. Entre 60 e $160 \mathrm{~cm}$ não há defasagem de tempo aparente para as temperaturas máximas. Para o dia 03 (fig.6), os valores máximos também como no dia 2 , foram atingidos a $1 \mathrm{~cm}$ do solo. Existe a mesma defasagem de tempo entre 1; 60 e $160 \mathrm{~cm}$ da superfície. As temperaturas de queima a 60 e $160 \mathrm{~cm}$ foram mais altas no dia $3 \mathrm{em}$ função da menor velocidade do vento $\left(14,4 \mathrm{~km} \cdot \mathrm{h}^{-1}\right.$ contra $16,2 \mathrm{~km} \cdot \mathrm{h}^{-1}$ no dia 3 ), embora a umidade relativa neste dia tenha sido ligeiramente maior (média de 59\% contra 53\% no dia anterior), no momento da queima. Estes valores foram semelhantes aos descritos por Vega (1996) em queimas controladas para umidade relativa do ar entre 25 e $75 \%$, porém mais altos com relação a velocidade do vento de 0,3 a 5,0 $\mathrm{km} . \mathrm{h}^{-1}$, na União Européia. Coutinho (1996) afirma que as temperaturas de queima de cerrado podem atingir $800^{\circ} \mathrm{C}$ ou mais, mas que esta elevação da temperatura é de curta duração. Considerando-se o tempo de permanência da temperatura acima de $60^{\circ} \mathrm{C}$ para o dia 3 na superfície do solo, observou-se uma permanência de 320 segundos, ou seja, bem superior ao encontrado por Miranda et al. (1996), que foi de 150 segundos. A diferença aí encontrada pode ser explicada pelas características do material combustível, o qual naquele trabalho constitui-se principalmente de combustíveis finos de rápida combustão, que não é o caso da bracatinga. Já as temperaturas máximas variaram de 604 a $752^{\circ} \mathrm{C}$ a $60 \mathrm{~cm}$ de altura, as quais ficaram dentro da faixa de variação normal para queimadas em cerrado (entre 70 e $800^{\circ} \mathrm{C}$ na superfície do solo e 200 a $800^{\circ} \mathrm{C}$ a cerca de 1 metro do solo) conforme citam Miranda et al. (1993) e Frost e Robertson (1987). 


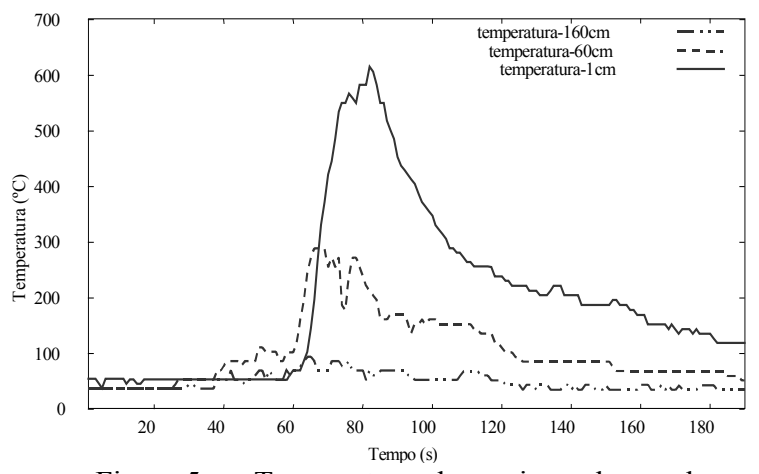

Figura 5: Temperatura de queima observada em três alturas, no dia 02/09/1998

Figure 5: Burning temperature at three height levels (02/09/1998)

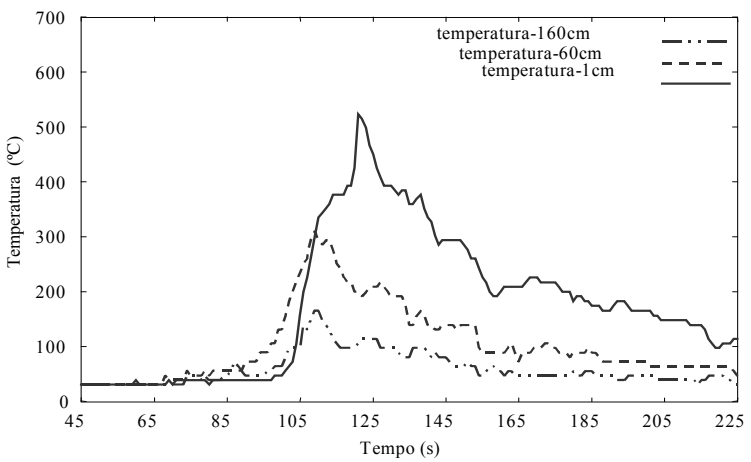

Figura 6: Temperatura de queima observada em três alturas, no dia 03/09/1998

Figure 6: Burning temperature at three height (03/09/1998)

Temperatura do solo: A temperatura que estava em torno de $23^{\circ} \mathrm{C}$ a $2,5 \mathrm{~cm}$ de profundidade, permaneceu assim até a passagem do fogo, elevando-se gradualmente até pouco acima dos $24^{\circ} \mathrm{C}$ aos 445 segundos, permanecendo neste patamar até 500 segundos, resultado que mostrou não haver alteração da temperatura que afete a atividade biológica do solo nesta profundidade. É de se esperar que em níveis mais superficiais haja alterações de temperatura mais evidentes, como as encontradas por Neves e Miranda (1996), onde a temperatura chegou a $52,8^{\circ} \mathrm{C}$ a $1 \mathrm{~cm}$ de profundidade. A umidade da manta ou serapilheira constituída em boa parte de folíolos de bracatinga, formou uma massa densa que funcionou como isolante térmico entre o fogo e o solo. Vega (1996) também demonstrou pouca variabilidade da temperatura num solo, quando a umidade da manta estava acima de $70 \%$.

\section{CONCLUSÕES}

i) $\mathrm{Na}$ área desmatada, em função da mudança da cobertura vegetal e coloração da superfície, o albedo sofreu variações, diminuindo após a queima e recuperando os valores com o repovoamento vegetal;

ii) $\mathrm{Na}$ área com bracatinga, o valor do albedo foi menor que o da área desmatada, estando dentro dos limites encontrados na bibliografia: 0,16 a 0,19 ;

iii) As temperaturas máximas de queima ficaram dentro da faixa normal citada na bibliografia, podendo nesta situação, até serem benéficas para a regeneração da bracatinga, cujas sementes necessitam de um choque térmico para superar a dormência;

iv) A duração da temperatura acima de $60^{\circ} \mathrm{C}$ (pulso de calor) foi o dobro da encontrada na região do cerrado;

v) A passagem do fogo sobre os resíduos da bracatinga pouco alterou a temperatura do solo, mostrando não haver grandes mudanças na atividade biológica do solo em função da temperatura de queima;

vi) Há necessidade de se repetir as medidas de temperatura em outras profundidades a partir da superfície, uma vez que a distribuição das sementes de bracatinga se dá em maior número nas primeiras camadas de solo.

\section{REFERÊNCIAS}

COUTINHO, L. M. O Cerrado e a ecologia do fogo. Ciência hoje. Rio de Janeiro, 12(68): 2230,1990.

DE BANO, L. F.; DUNN, P. H.; CONRAD, C. E. Fires effect on physical and chemical properties of chaparral soils. In: SYMPOSIUM ON ENVIRONMENTAL CONSEQUENCES OF FIRE AND FUEL MANAGEMENT IN MEDITERRANEAN ECOSSYSTEMS, Washington-D.C. U.S.D.A. Forest service, 1977. p. 65-74. (Gen. Tech. Rep. WO-3).

DIAS, I. O. F.; MIRANDA, A. C.; MIRANDA, H. S. Efeito das queimadas no microclima de solos de campos de Cerrado DF/Brazil. In: SIMPÓSIO IMPACTO DAS QUEIMADAS SOBRE OS ECOSSISTEMAS E MUDANÇAS GLOBAIS. CONGRESSO DE ECOLOGIA DO BRASIL, 3, 1996. Anais... Brasília, DF., 1996, p.11-19. 
DIAS, N. L.; OKAWA, C. M. P.; GRODZKI, L. et al. Os experimentos intensivos de campo dos estudos de mesoescala para o lago de Itaipú (EXINC/MESOLIT).In: CONGRESSO BRASILEIRO DE METEOROLOGIA, 10, Brasília, DF, 1998. Anais... Brasília: 1998.

FROST, P. G.; ROBERTSON, F. The ecological effects of fire in savanna. In: Determinants of Tropical Savannas. B.H. Walber (ed.) Oxford: IRL Press Limited. 1987. p.93-141.

GIELOW, R.; ALVALÁ, R. C. S.; HODETT, M.G. et al. Albedo no pantanal sulmatogrossense durante e após o período de inundação de 1998. In: CONGRESSO BRASILEIRO DE AGROMETEOROLOGIA, 11 (1999); REUNIÃO LATINOAMERICANA DE AGROMETEOROLOGIA., 2 (1999). Florianópolis: SBA n.1, 1999. CD-ROM.

ITO, M.; IZUMI, S. Temperatures during grassland fires and their effect on some species in Kawatabi, Miyagi Prefecture. Tokyo Univ. Sci. Rep. Res. Inst., v. D-11, n.2, p. 109-114. 1960.

MIRANDA, A. C.; MIRANDA, H. S.; DIAS, I. de F.O. et al. Soil and air temperatures during prescribe cerrado fires in Central Brazil. Journal of Tropical Ecology, v.9, n.3, p.313320, 1993.

MIRANDA, H. S.; ROCHA E SILVA, E. P.; MIRANDA, A. C. Comportamento do fogo em queimadas de campo sujo. In: SIMPÓSIO IMPACTO DAS QUEIMADAS SOBRE OS ECOSSISTEMAS E MUDANÇAS GLOBAIS. CONGRESSO DE ECOLOGIA DO BRASIL, 3, Anais... Brasília-DF: 1996. p.1-9.

MONTEITH, J. L. Priciples of Environmental Physics. London: Edward Arnold Publishers, 1973. 241p.

NEVES, B. M. C.; MIRANDA, H. S. Efeitos do fogo no regime térmico do solo de um campo sujo de cerrado. In: SIMPÓSIO IMPACTO DAS QUEIMADAS SOBRE OS ECOSSISTEMAS E MUDANÇAS GLOBAIS. CONGRESSO DE ECOLOGIA DO BRASIL, 3, Brasília, 1996. Anais... Brasília-DF: 1996. p.20-36.
OKAWA, C. M. P.; GRODZKI, L.; DIAS, N. L. et al. Medições de fluxos de energia com métodos tradicionais alternativos sobre uma cultura de soja em Santa Therezinha de Itaipu, PR. In: CONGRESSO BRASILEIRO DE METEOROLOGIA, 10, Brasília, 1998. Anais...Brasília-DF, 1998. p.40-56.

OKAWA, C. M. P.; DIAS, N. L.; GRODZKI, L. et al. Resultados de um experimento micrometeorológico em uma cultura de soja. In: SIMPÓSIO BRASILEIRO DE RECURSOS HÍDRICOS, 12, Belo Horizonte, 1999. Anais... Belo horizonte, 1999.

RALSTON, C. W.; HATCHELL, G. E. Effects of prescribed burning on physical properties of soil. In: PRESCRIBED BURNING SYMPOSIUM, 1971. Charleston, South Carolina. Proceedings... U.S.D.A. Forest Service, 1971. p. 68-84.

ROSE, C. W. Agricultural Physics. Pergamon Press: London, 1969.p.30-88.

SONG, J. Diurnal asymmetry in surface albedo. Agricultural and Forest Meteorology, v.92, p.181-189, 1998.

VEGA, J. A. Investigation sobre control de incendios em España. In: SOARES, R.V.. REUNIÃO TÉCNICA CONJUNTA FUPEF/SIF/IPEF, 4 (1996); CURSO DE ATUALIZAÇÃO EM CONTROLE DE INCÊNDIOS FLORESTAIS, 2 (1996). Anais... Curitiba: FUPEF/DSM-SCA-UFPR, 1996.

WRIGHT, H. A. BAILEY, A. W.. Temperature and heat effects. In: Fire ecology. United States and Canada. N. York: John Wiley \& Sons, 1982, p.8-23 